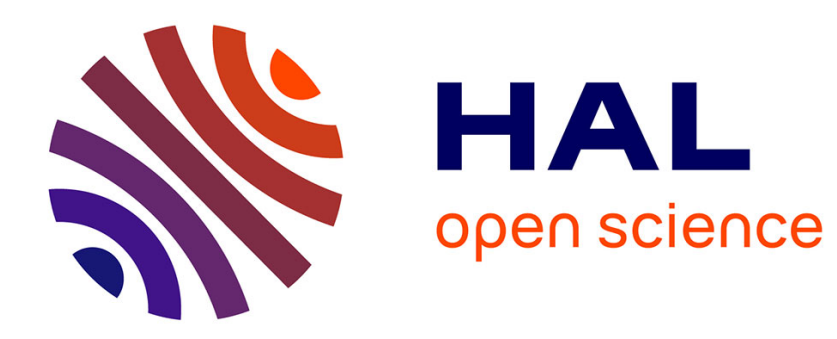

\title{
Questions de saillance et épigraphie maya
}

Jean-Michel Hoppan

\section{To cite this version:}

Jean-Michel Hoppan. Questions de saillance et épigraphie maya. Faits de langues, 2012, La saillance (39), pp.117-127. halshs-00728394

\section{HAL Id: halshs-00728394 https://shs.hal.science/halshs-00728394}

Submitted on 5 Sep 2012

HAL is a multi-disciplinary open access archive for the deposit and dissemination of scientific research documents, whether they are published or not. The documents may come from teaching and research institutions in France or abroad, or from public or private research centers.
L'archive ouverte pluridisciplinaire HAL, est destinée au dépôt et à la diffusion de documents scientifiques de niveau recherche, publiés ou non, émanant des établissements d'enseignement et de recherche français ou étrangers, des laboratoires publics ou privés. 


\section{Questions de saillance et épigraphie maya}

Jean-Michel Hoppan*

\section{1) INTRODUCTION}

Dans le domaine des langues amérindiennes, la famille maya est celle qui a laissé le plus de traces écrites antérieures à l'arrivée des Européens et aux profonds bouleversements subis depuis par la plupart de ces langues.

Dès le début de la seconde moitié du Ier millénaire avant notre ère, une écriture logo-syllabique (dite communément en "glyphes") fut en effet élaborée par les Mayas ${ }^{1}$ afin de transcrire leurs propres langues, et ainsi commencer à écrire leur propre histoire. Délaissée depuis plus de trois siècles pour avoir été remplacée par l'alphabet latin, l'écriture maya fut utilisée durant environ 2000 ans, valant aux langues mayas d'être celles qui, de toute l'Amérique, nous ont légué le plus grand corpus de textes antérieurs au XVIème siècle.

On considère que, dans le cadre des écritures américaines autochtones, l'écriture logo-syllabique maya fut au moins l'une des plus performantes. Bien que le déchiffrement n'en ait aujourd'hui pas complètement abouti, de grands progrès ont récemment été effectués. Ces avancées ont mis en évidence que cette écriture était apte à transcrire efficacement la plupart des unités lexicales (y compris les verbes) et morphèmes grammaticaux des langues mayas. Ceci n'était pas, au regard des connaissances actuelles, le cas dans la majorité des autres écritures de l'Amérique précolombienne.

A priori, ce constat semblerait rendre le domaine maya plus favorable que ses homologues à un essai sur la saillance dans les états pré-modernes de langues amérindiennes.

Néanmoins, cet aspect plus opportun ne doit pas cacher que ces occasions restent toutes relatives, et ce compte tenu de quelques conditions qui restreignent les possibilités d'explorer profondément ce sujet. Voici les trois principales :

Premièrement, le déchiffrement de l'écriture maya n'est non seulement pas encore achevé mais, notamment faute d'avoir découvert quelque véritable "document-clé" équivalent à ce que la Pierre de Rosette fut pour les hiéroglyphes égyptiens, il faut garder à l'esprit que ce qui est aujourd'hui déchiffré l'est en grande partie de façon hypothétique. Dans le millier de signes actuellement identifiés, des lectures ont en effet été établies pour un peu plus de 200

\footnotetext{
* SeDyL-CELIA (UMR 8202), CNRS, INALCO, IRD. Courriel : hoppan@vjf.cnrs.fr

1 On entend là par "Mayas" les locuteurs des langues appartenant à la famille maya. Parmi ces langues, celle que l'on parle encore aujourd'hui dans l’ancien pays du Yucatán -qui correspond approximativement aux actuels Etats mexicains de Yucatán, Campeche et Quintana Roo- est la seule à autrefois s'être appelée la langue maya, ou maya t'aan. De nos jours, elle est de plus en plus souvent appelée le yucatèque, afin de la différencier de la vingtaine de langues apparentées que par extension on a également qualifiées de mayas.
} 
logogrammes ${ }^{2}$ et environ 150 phonogrammes $^{3}$. Il reste donc rare de pouvoir effectuer des transcriptions à la fois complètes et indiscutables des textes actuellement connus dans cette écriture.

Deuxièmement, les scribes mayas n'avaient pas pour habitude, même si l'écriture en glyphes le permettait, de transcrire complètement l'aspect grammatical d'un texte. Les abréviations étaient fréquentes, même dans la notation des unités lexicales, et il n'était pas rare de ne recourir à une transcription complète que lorsque cela pouvait lever une ambigüité ou par recherche esthétique, sachant que chez les anciens Mayas l'écriture n'était pas qu'une simple transposition graphique du langage car la calligraphie était en même temps un genre majeur d'art plastique. Si les morphèmes grammaticaux actuellement identifiés sont pour l'essentiel les affixes de flexions verbale et nominale, des agentifs, des suffixes tels que locatifs et marqueurs d'abstraction ou de possession inaliénable, ainsi que quelques prépositions, conjonctions, marques de pluriel, classificateurs et négations, on ne trouve pratiquement pas de déterminants, déictiques ${ }^{4}$ ou marques d'aspect, bien que ce dernier est fondamental dans la grammaire des langues mayas.

Troisièmement, le contenu de ce qui nous est parvenu des écrits en glyphes mayas est lui-même très particulier et volontiers propice à l'emploi d'un langage occulte et hermétique. Les écrits sur papier d'écorce ${ }^{5}$ consistent tous en des manuscrits tardifs ${ }^{6}$ qui apparaissent comme des sortes de recueils d'almanachs divinatoires. Le reste est essentiellement représenté par quelques milliers de textes à caractère mythico-historique, gravés à l'époque classique ${ }^{7}$ dans la pierre de nombreux monuments, et par quelques autres milliers d'inscriptions (de datation comparable) sur du matériel mobilier, en particulier sur des céramiques historiées. Habituellement, ces dernières portent d'une part les formules dédicatoires mentionnant en particulier les nom et titres du commanditaire de

\footnotetext{
2 Parmi les mayanistes, on appelle logogramme des signes qui transcrivent chacun une (ou plusieurs) unité(s) lexicale(s). Les logogrammes apparaissent ainsi comme des signes investis d'une charge sémantique.

3 Dépourvus de relation intrinsèque au sens, ces phonogrammes notaient de l'ordre de 80 syllabes, sachant cependant qu'il subsiste toujours quelques vides dans le syllabaire maya, tel qu'il a pu de nos jours être reconstitué, tandis que certaines syllabes (dans le cadre de ce que l'on désigne sous le nom d'allographie) pouvaient être marquées de plusieurs façons différentes.

${ }^{4}$ Les langues mayas possèdent pourtant un riche répertoire de déictiques.

5 Le papier d'écorce fut manifestement, jusqu'à l'époque de la conquête espagnole, le support de la plus grande partie de la production écrite maya. En raison des dommages du temps (conditions climatiques régnant dans la plupart des régions habitées par les Mayas et autodafés des Espagnols, notamment), cette production a presque entièrement disparu, à l'exception de quelques manuscrits très rares, dits codex.

6 Aucun de ces manuscrits ne semble être antérieur à la phase récente de l'époque postclassique, à partir du XIIIème siècle.

7 L'époque dite maya classique s'est écoulée entre le IIIème et le Xème siècles de notre ère, selon la corrélation entre calendriers maya et chrétien la plus largement admise aujourd'hui.
} 
l'objet et, d'autre part, des légendes iconographiques destinées à commenter les scènes, en général mythologiques, qui étaient peintes ou gravées sur ces objets.

On ne dispose donc d'aucun document qui aurait éventuellement traité de la vie de tous les jours dans un parler courant. On ne connaît pas non plus de roman, ni d'essai philosophique, correspondance ou tout autre genre de production écrite ; pas même les brouillons ou exercices à caractère scolaire que présuppose pourtant l'existence d'une écriture.

Nonobstant ce constat, on s'est proposé d'examiner ce qui pourrait permettre de nourrir des réflexions sur la saillance dans le corpus actuellement connu des textes mayas de l'époque préhispanique. On résume ici quelques résultats de ce travail, en soulignant toutefois qu'il convient de garder à l'esprit que cet essai est le reflet d'une étude à son commencement, une introduction non exhaustive à de possibles approfondissements ultérieurs pour proposer un point provisoire sur la question.

Ainsi, on ne dispose pratiquement de rien qui puisse nous éclairer en ce qui concerne des aspects de la saillance tels que la hiérarchie des termes selon leur caractère animé ou inanimé, ou bien humain ou non-humain. Rien non plus sur le marquage des indices personnels, et trop peu sur l'incorporation nominale.

En ce qui concerne les marques de genre, on ne dispose aussi que de très peu de données. Ces marques se résument pour ainsi dire à un seul élément, qui n'apparaît que dans les noms et titres des femmes afin de les distinguer des noms et titres d'hommes, qui ne le portent pas. Il s'agit d'un signe antéposé, figurant une tête de femme et qui selon les cas $^{8}$ était lu $i x$-, agentif féminin, IXIK "dame ", ou peut-être aussi $N A$ ' "mère ", ainsi que le suggère la valeur syllabique na adoptée quand il était employé comme phonogramme.

Même les déictiques semblent quasiment absents des inscriptions mayas ${ }^{9}$, bien que (comme on l'a mentionné plus haut) les langues mayas sont riches de ces particules, permettant d'exprimer par exemple la distinction entre ce qui est près et ce qui est loin, ce qui est connu ou ce qui ne l'est pas etc.

On est en revanche mieux pourvu en ce qui concerne quelques autres aspects de la problématique, tels que la question des classificateurs numéraux, morphèmes grammaticaux dont une fonction primordiale est manifestement de rendre saillant en tant qu'individu ou unité comptable un élément nommé. De même à propos de ce qui est relatif, dans la flexion verbale, à l'emploi des "série A" / "série B"10 et diverses voix actuellement reconnues dans les inscriptions, la

8 Ce sont en général le contexte ou les compléments phonétiques qui déterminent les lectures. Ces dernières peuvent également avoir évolué et varié selon les lieux.

9 On n'en retrouve que de fort rares traces. C'est par exemple le cas dans un système de pronoms indépendants, où la 1ère personne «moi » est marquée par hiin (de ha'een: particule $h a^{\prime}+\mathrm{B} 1$-een, 1ère personne de la série des pronoms absolutifs), où la 2e « toi » est ha'at (ha' + B2 -at) et la 3e « lui/elle » ha'i (ha' + B3 -0 + i, cette dernière particule pouvant être interprétée comme un déictique dans une forme que l'on pourra aussi traduire par « celui/celle-ci » ou « celui/celle-là »).

10 Les langues mayas sont pour la plupart des langues dites à ergativité "scindée", où les pronoms personnels marquant l'ergatif sont préfixés au lexème fléchi (pronoms de la "série A") tandis que ceux de l’absolutif sont des suffixes (pronoms de la "série B"). 
variabilité de l'attribution du prédicat rendant selon les cas un terme plus remarquable qu'un autre.

\section{2) LES CLASSIFICATEURS NUMÉRAUX}

Dans les langues mayas, ce que l'on appelle les classificateurs numéraux ${ }^{11}$ sont des particules postposées au nombre lorsqu'il s'agit d'exprimer une quantification. Autrefois très riche, le système des classificateurs numéraux tend, dans les langues mayas contemporaines, à un appauvrissement considérable. Cet appauvrissement est certainement lié au fait que la numération décimale espagnole, difficilement compatible avec la numération vigésimale des Mayas, a pratiquement éclipsé cette dernière (à l'exception des plus petits nombres, en général pas au-delà de 5). En yucatèque par exemple, la langue courante n’a plus guère qu'un classificateur pour les humains (-tuul) et un autre pour tout le reste $(-p$ 'eel) : on pourra dire ox-tuul winik pour "trois hommes » mais on dira $o x$ p'eel waaj pour «trois tortillas ${ }^{12}$. Les textes alphabétiques anciens montrent cependant bien que le système était en effet d'une grande richesse auparavant, avec tout un répertoire de classificateurs variant par exemple selon la forme des objets à quantifier, s’ils étaient plats, gros ou ronds etc.

Depuis quelques années, on est parvenu à identifier dans les inscriptions glyphiques certains de ces morphèmes grammaticaux, pour l'instant pas plus d'une dizaine. A priori, on est donc encore loin d'avoir retrouvé dans les écrits des temps préhispaniques toute la richesse du système de classificateurs, d'autant que comme tant d'autres éléments grammaticaux ils n'étaient que rarement marqués. La plupart du temps, les classificateurs numéraux étaient en effet sousentendus, abrégés ou éludés. La raison principale en est probablement que, comme on l'a suggéré précédemment, le propos principal de l'écriture en glyphes n'était pas de se restreindre à une transposition graphique de la langue parlée mais qu'il y avait aussi par le biais de l'image d'autres informations à exprimer. L'art de la calligraphie maya permettait ainsi aux scribes de très volontiers faire l'économie de la notation des classificateurs si, au final, le contexte allait permettre au lecteur de comprendre sans ambiguïté ce qu'il fallait.

Un des classificateurs numéraux mayas les mieux attestés dans les glyphes est la particule -tal, dont on illustre en cotexte un exemple tiré de l'inscription de la Stèle 10 de Seibal :

11 Selon Colette Grinevald (1999:111), les dits classificateurs numéraux sont, parmi les trois principaux types de classificateurs identifiés en linguistique, «le type le plus commun et le mieux établi ».

12 Il est sans doute intéressant de remarquer que, dans cet appauvrissement, continue à être distingué avant tout ce qui est humain : la classe qui relève de l'humanité paraît en effet plus remarquable que toutes les autres classes, en somme plus saillante. 

(1) jo'-ajaw ox[-te]-k'anasiiy u-hun-tal-winikhaab-0
5-Ahau 3[-CLAS.]-Kayab A3-1-CLAS.katun-B3
'[Le] 5 Ahau 3 Kayab, (c’est) le $1^{\text {er }}$ katun ("venu").'

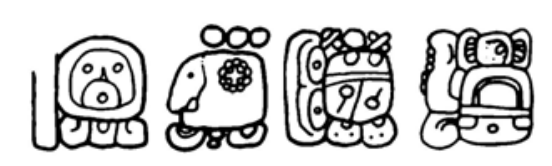

Figure 1 : Stèle 10 de Seibal (El Petén, Guatemala), 849 AD,

glyphes B1 à A3 (d'après dessin de I. Graham 1996, 7:32)

Cet exemple permet de constater que, comme dans les langues modernes, certains classificateurs peuvent être mis en relation avec des bases verbales, ici la racine du verbe $\operatorname{tal}(\mathrm{el})$ « venir(/arriver) ».

Ceci n'est toutefois pas le cas du classificateur que l'on retrouve le plus fréquemment dans les inscriptions de l'époque classique. Ce classificateur est te, homophone du lexème qui signifie « bois /arbre» dans les langues mayas le plus souvent attestées pour l'époque classique. Il semble qu'il s'agissait d'un classificateur fort générique, bien qu'en yucatèque son usage concerne surtout le comput. Le dictionnaire du XVIème siècle dit Calepino de Motul en donne effectivement deux définitions : la première dit " cuenta para anyos y para los dias de los meses y para leguas » 13 , la seconde dit « cuenta para cacaos, huevos y calabazas » ${ }^{14}$ (Arzápalo Marín 1995: 706 et 2105).

On en illustre deux exemples provenant d'une inscription du Yucatán (Panneau 2 de Xcalumkin), dans un usage qui semble tout à fait correspondre à la première définition (avec un décompte de périodes positionnant à partir du début du grand cycle actuel du calendrier maya une date dans le cycle solaire) :
(2) bolon-pih jo'lajuun-winikhaab ka'lajuun-te-haab

9-baktun 15-katun 12-CLAS.-tun jour
'9 baktun (/ "périodes de 400 années (de compte)"), 15 katun (/ "périodes de 20 années (de compte)"), 12 tun (/ "années (de compte)"), 6 uinal (/ "mois de 20 jours") (et) 9 jours'

13 « classificateur pour compter les années, les jours des mois et les lieues » (traduction de J-M. Hoppan).

14 « classificateur pour compter le cacao, les œufs et les courges » (traduction de J-M. Hoppan). 


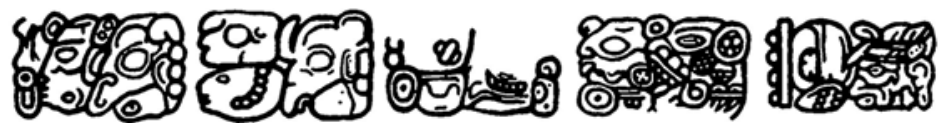

Figure 2 : Panneau 2 de Xcalumkin (Campeche, Mexique), 743 AD,

glyphes 2 à 6 (d'après dessin de E. Von Euw (Graham \& Von Euw 1992, 4:180))

(3) ti-hun-te-k'ank'iin

PRÉP.-1-CLAS.-Kankin

'le 1 Kankin (le "premier" jour du mois de 20 jours appelé Kankin)'

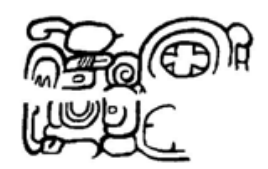

Figure 3 : Panneau 2 de Xcalumkin (Campeche, Mexique), 743 AD,

glyphe 13 (d'après dessin de E. Von Euw (Graham \& Von Euw 1992, 4:180))

On observe que le classificateur est bien marqué pour toutes les unités à l'exception de celles qui sont supérieures à l'année de compte dite tun (donc à l'exception du katun et du baktun), ainsi que dans la notation du nom du jour dans le calendrier solaire.

Mais ce classificateur -te, du moins dans les nombreuses inscriptions provenant d'autres régions que le Yucatán, paraît avoir été utilisé d'une façon beaucoup plus large encore que les deux usages mentionnés pour le yucatèque ancien. C'est ce que montre un glyphe tel que celui de ox-te tun, nom ancien de la cité de Calakmul qui signifiait littéralement " (les) trois pierres », constituant une référence au foyer primordial de la création (archétype du foyer maya traditionnel, composé de trois pierres). On en illustre une occurrence gravée sur un panneau provenant de Cancuen, dans son cotexte :

(4) yuknoom-ch'een $\begin{array}{ll}\text { ox-te-tun } & \text { kaloom-te } \\ \text { 3-CLAS.-pierre } & \text { "kaloomte" }\end{array}$ Yuknoom Ch'een

'Yuknoom Ch'een, (le) "kaloomte" (= grand roi / empereur) des trois pierres' 


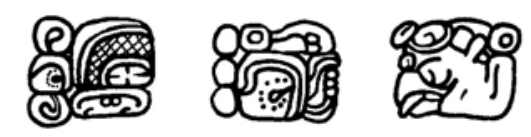

Figure 4 : Panneau 1 de Cancuen (Guatemala), ca $773 \mathrm{AD}$,

glyphes C8 à C9 (d'après dessin de S. Gronemeyer 2005)

Un tel exemple, de même qu'en fait de nombreux autres, conduit volontiers le chercheur à s'interroger sur la fonction fondamentale du dit classificateur numéral dans les langues mayas. En effet, on peut pour le coup se demander si sa raison essentielle n'était pas avant tout de rendre quantifiable, en le discrétisant, ce qui conceptuellement était continu ${ }^{15}$, plutôt que de servir à y effectuer une classification (ainsi que le nom donné par les linguistes à ces particules tendrait à le faire penser). Il paraît cependant clair que la notion de saillance s’y manifeste au moins à travers une individuation sémantique.

\section{2) LA FLEXION VERBALE}

De façon plus déterminante que par les temps, la flexion des verbes est profondément marquée dans la majorité des langues mayas par l'aspect, accompli ou inaccompli, ainsi que par une ergativité "scindée" où l'ergatif est associé aux verbes transitifs, tandis que l'absolutif est lié aux verbes intransitifs 16 , le mode indicatif étant caractérisé par l'emploi des voix active, passive, anti-passive et médio-passive (dite aussi moyenne). Ainsi qu'on l'a vu précédemment, l'aspect n’est curieusement pas marqué dans les inscriptions ${ }^{17}$ mais la flexion ergatif $v s$ absolutif et l'emploi des quatre voix de l'indicatif ont en revanche bien été identifiés, permettant au linguiste d'observer les modalités de changement d'une voix à une autre et d'ainsi pouvoir éventuellement déterminer dans quelle mesure cette variabilité rend selon les cas un mot ou un syntagme plus saillant qu'un autre dans une même phrase ou proposition. On présente ici trois cas de telles variations sur des formules relativement bien documentées, pris dans l'abondant corpus des inscriptions monumentales de l'époque classique: il s'agit de la "fin de katun" en tant qu'indication d'accomplissement d'une période majeure dans le calendrier, la "fin de katun"

15 En l'occurrence ici, « la (substance) pierre » devient « des pierres (dénombrables) ».

16 En association aux substantifs, l'absolutif est en outre équivalent au verbe « être » qui n'existe pas en maya (dans son sens hispanique de ser).

17 Il pourrait en fait avoir été marqué d'une autre façon : l'aspect des glyphes verbaux semblerait par défaut être systématiquement accompli (d'où l'absence de nécessité d'une notation explicite), tandis que des inaccomplis de type «il/elle est en train de ... » pouvaient être exprimés par l'emploi absolutif d'un syntagme nominal, littéralement traduisible par « (c'est) son image en ... ». Une telle déconnexion entre grammaire de la langue et "grammaire" de l'écriture peut éventuellement être liée au caractère très figuratif qu'a toujours conservé l'écriture maya. 
en tant que célébration de l'accomplissement de cette période par une cérémonie, effectuée sur un monument, et la "réception/prise du (sceptre) Kauil", expression de l'avènement d'un roi.

3.1) La "fin de katun", indication de l'accomplissement d'une période

Sur des stèles de même époque et témoignant de son emploi à l'absolutif, cette formule apparaît volontiers aux voix passive ou médio-passive. Le texte de la Stèle B de Copán fournit un exemple caractéristique du passif :

(5) tsuuts-aj-0 jo'lajuun-winikhaab

terminer-PAS.-B3 15-katun

'est terminé [le] 15[ $\left.{ }^{\mathrm{ème}}\right]$ katun'

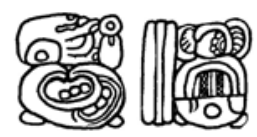

Figure 5 : Stèle B de Copán (Honduras), 731 AD,

glyphes B5 et B6 (d'après dessin de Dowd \& Fash (Fash 1991: 30 fig.17))

Une inscription de la Stèle 16 de Tikal fournit une occurrence de la même formule à la voix médio-passive :

(6) tsuuts-uuy-0

$$
\text { u-chanlajuun-winikhaab }
$$

terminer-MOY.-B3

A3-14-katun

's'est terminé le $14^{\text {ème }}$ katun'

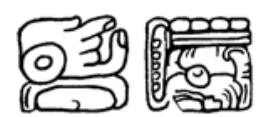

Figure 6 : Stèle 16 de Tikal (El Petén, Guatemala), 711 AD,

glyphes A3 et A4 (d'après dessin de W. Coe (Jones \& Satterthwaite 1982: fig.22))

Rien ne permet de supposer à travers ces exemples que la différence de voix ait induit une différence sémantique. L'ensemble de l'échantillon analysé dans son contexte montre que, dans les deux cas, il s'agit en général de propositions simples, éventuellement suivies d'un complément circonstanciel de lieu et d'une expression du type "sous les auspices de + nom propre".

3.2) La "fin de katun", célébration de l'accomplissement d'une période

Indiquant le même évènement que précédemment dans un sens toutefois distinct et par des signes différents, cette formule paraît sujette à de plus amples 
variations grammaticales. La Stèle 15 de Copán en fournit un exemple transitif, fléchi selon le paradigme verbal ergatif ${ }^{18}$ :
(7) u-k'aal-aw
tuun ?-kin-ich
$k^{\prime} u k^{\prime}-m o$ '
A3-clore-TRANS
pierre ?-soleil-visage
[Yax] K’uk' Mo'
'[le] ? roi Yax K'uk’ Mo' a ceint (le) mégalithe
(= a célébré la commémoration du katun)'

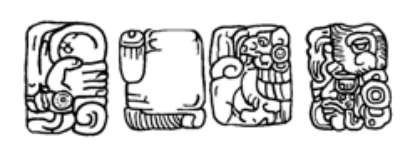

Figure 7 : Stèle 15 de Copán (Honduras), 524 AD,

glyphes H2 à G4 (d'après dessin de L. Schele 1990: 23 fig.4)

La Stèle $\mathrm{C}$ de Quiriguá témoigne d'une occurrence où le verbe et son objet sont devenus un syntagme verbo-nominal intransitif, fléchi au passif :

(8) ox-k'aal-aj-0-tuun ? / ox-k'aal-tuun-aj-0 ?

3-clore-PAS.-B3-pierre ? / 3-clore-pierre-PAS.-B3 ?

'trois (sont les) ceinte(s) pierre(s) ? / trois (est le) ceinte-pierre ?

(= les trois pierres sont ceintes / le trois-pierres est ceint)'

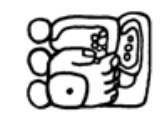

Figure 8 : Stèle C de Quiriguá (Izabal, Guatemala), 775 AD,

glyphe A7 (d'après dessin de M. Looper (Schele \& Looper 1996: 92))

La même inscription fournit aussi des exemples à l'absolutif :

(9) i(yuwal)-uut-iiy-0 k'aal-tuun-0 na(aj)-itsam-? puis-survenir-PRÉT.-B3 clore-pierre-B3 $1^{\mathrm{er}}$-"oiseau Itzamna"

'(et) puis il était arrivé que (est) ceinte-pierre (le) premier oiseau Itzamna

(= le premier oiseau Itzamna a ceint la pierre)’

18 A la différence de celle-ci, un seul exemple à l'ergatif est connu pour la formule précédente d'indication de l'accomplissement d'une période, sur la Stèle 31 de Tikal (époque classique ancienne, $445 \mathrm{AD}$ ) : « il a terminé [le] 18[ $\left.{ }^{\mathrm{e} m e}\right]$ katun, sous les auspices du seigneur/maître des katun Yax Nuun Ayiin I ». 


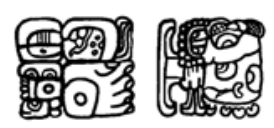

Figure 9 : Stèle C de Quiriguá (Izabal, Guatemala), 775 AD,

glyphes A12 à B12 (d’après dessin de M. Looper (Schele \& Looper 1996: 92))

Le texte de l'Autel 21 de Caracol donne un autre exemple à l'absolutif, où le syntagme est fondamentalement devenu nominal et prend la place de prédicat de l’énoncé :
(10) u-k'aal-tuun-0
uuk-ajaw
7-Ahau
ox-uniw
A3-clore-pierre-B3
3-Kankin
'(c'est) la ceinte-pierre (= commémoration du katun) du 7 Ahau 3 Kankin'

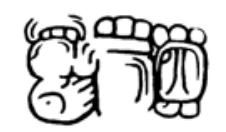

Figure 10 : Autel 21 de Caracol (Belize), 593 AD,

glyphes A'1 à B'1a (d'après dessin de S. Houston (Schele \& Freidel 1990: 172 fig.5:4))

La Stèle 7 de Copán fournit une autre occurrence où le syntagme est fondamentalement devenu nominal, à partir d'un passif :

(11) ox-te-suuts'

3-CLAS.-Zodz

u-k'aal-aj-0-tuun ? / u-k'aal-tuun-aj-0 ?

A3-clore-PAS.-B3-pierre ? / A3-clore-pierre-PAS.-B3 ?

'[le] 3 Zodz, (c'est) sa ceinte-pierre (= commémoration du katun)'

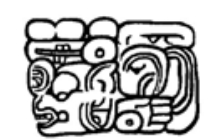

Figure 11 : Stèle 7 de Copán (Honduras), 613 AD,

glyphe B6 (d'après dessin de L. Schele et D. Stuart (Schele 1990: 28 fig.9))

La seule constante observée là, dans l'échantillon analysé en contexte, est que l'emploi verbal à l'ergatif ( $c f$ Figure 7) n'est employé que lorsque le sujet est le 
nom d'un roi, comme si cette formulation était réservée à l'expression de la cérémonie lorsqu'elle était publiquement effectuée par un souverain, sans cependant que cela en soit exclusif car pour autant d'autres tournures pouvaient être utilisées dans ce cas. Il semblerait aussi que les formes verbales absolutives soient plus fréquentes lorsque le sujet est le nom d'une divinité, sans toutefois que cela soit systématique. L'absence d'une différenciation catégorique tient peut-être au fait que les souverains mayas classiques étaient vus comme des rois divinisés. Il est également possible que l’emploi des formes nominalisées ait été destiné à rendre remarquable l'évènement relaté, alors que les formes verbales rendraient plutôt frappant le nom des acteurs de cet évènement, la solution choisie pouvant par conséquent faire apparaître tel ou tel mot ou syntagme comme plus saillant qu'un autre. 
3.3) La" prise de Kauil", avènement royal

Indiquant un évènement qui par définition est de nature plus explicitement politique, cette formule où le sujet est toujours le nom d'un roi apparaît également fléchi de façons diverses. La Stèle $\mathrm{F}$ de Quiriguá en porte une occurrence à l'anti-passif :

(12) k'am-aaw-k'awiil-0 ? / k'am-k'awiil-aw-0 ? recevoir-ANT.-K'awiil-B3 ? / recevoir-K'awiil-ANT.-B3 ?

k'ak'-tiliw-chan-yopaat

K'ak' Tiliw Chan Yopaat

'K’ak' Tiliw Chan Yopaat a reçu/pris-K’awiil (= accédé au pouvoir)'

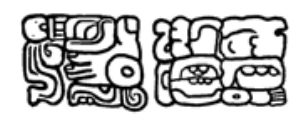

Figure 12 : Stèle F de Quiriguá (Izabal, Guatemala), 761 AD,

glyphes B6 et A7 (d'après dessin de M. Looper (Schele \& Looper 1996: 124))

L'inscription d'un monument voisin (Stèle E de Quiriguá) en fournit un exemple à l'absolutif, où le syntagme est fondamentalement devenu nominal :

(13) u-k'am-k'awiil-0

A3-recevoir-K'awiil-B3

k'ak'-tiliw-chan-yopaat ch'ajoom

K'ak' Tiliw Chan Yopaat ch'ajoom

'(c'est) la réception/prise-K'awiil (= accession au pouvoir) de K'ak' Tiliw Chan Yopaat, (le) ch'ajoom'

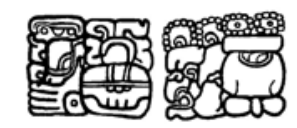

Figure 13 : Stèle E de Quiriguá (Izabal, Guatemala), 771 AD,

glyphes A8 et B8 (d'après dessin de M. Looper (Schele \& Looper 1996: 124))

Sur la Stèle J de Copán, un autre emploi à l’absolutif montre que le syntagme peut aussi rester fondamentalement verbal :

(14) k'am-iiy-k'awiil ? / k'am-k'awiil-iiy? recevoir-PRÉT.-K'awiil ? / recevoir-K'awiil- PRÉT. ?

k'in-ich yax-k'uk'-mo' 
soleil-visage Yax K'uk’ Mo'

'(le) roi Yax K’uk’ Mo’ est reçu/pris-K’awiil (= a accédé au pouvoir)'

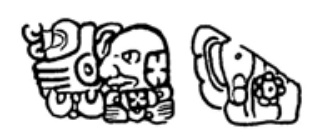

Figure 14 : Stèle J de Copán (Honduras), 702 AD,

glyphes Est 20 et 21 (d'après dessin de L. Schele (Schele \& Looper 1996: 101))

Là encore, l'emploi des formes nominalisées a peut-être eu pour fonction de rendre plus frappant ou remarquable l'évènement relaté que le nom du personnage impliqué.

\section{4) EN GUISE DE CONCLUSION}

Bien entendu loin d'être exhaustif, cet essai n'a pour ambition qu'un état des lieux provisoire. Il resterait encore à explorer d'autres aspects de la syntaxe des glyphes, tels que l'ordre des mots. Il suffit cependant à rendre compte de la difficulté d'aborder le thème de la saillance avec ce type de sources.

Les données les moins rares concernent essentiellement les dits "classificateurs numéraux" et la flexion verbo-nominale. Dans le premier cas, cette rareté empêche actuellement d'en tirer des résultats définitifs et tend surtout à laisser penser que, plus que des particules ayant pour fonction de classer, ces morphèmes servaient avant tout de marqueurs d'individuation pour rendre saillant en tant qu'élément comptable ce qui fondamentalement est continu. On rejoindra là les réflexions d’Alexandre François (1999), montrant que les classificateurs ont au plus un effet classifiant mais que leur supposée fonction de classifier n'existe de toute évidence pas. Dans le second cas, les observations effectuées pourraient suggérer d'une part que l'emploi d'une forme transitive, exprimée en maya par un verbe fléchi à l'ergatif, était réservé aux cas où le sujet est le nom d'un personnage ayant réellement existé (même s'il s'agit d'un roi divin, humain lieutenant des forces sacrées de l'univers parmi les autres humains), et d'autre part que l'utilisation de formes nominalisées était peut-être plus liée à une manifestation de saillance portant sur l'action/évènement, que sur ses acteurs.

\section{LISTE DES ABRÉVIATIONS JUXTALINÉAIRES :}

ANT. = anti-passif

A3 = pronom ergatif de série $A$, personne 3

$\mathrm{B} 1=$ pronom absolutif de série $\mathrm{B}$, personne 1

$\mathrm{B} 2$ = pronom absolutif de série $\mathrm{B}$, personne 2 
B3 = pronom absolutif de série $B$, personne 3

CLAS. = classificateur (numéral)

MOY. = médio-passif (voix moyenne)

PAS. $=$ passif

PRÉP. = préposition

PRÉT.. prétérit

TRANS. = suffixe de transitivité 
RÉFÉRENCES

ARZÁPALO MARÍN, Ramón

1995 Calepino de Motul. Diccionario Maya-Español, Universidad Nacional Autónoma de México, Mexico

FASH, William L.

1991 Scribes, Warriors and Kings: The City of Copan and the Ancient Maya, Thames and Hudson, Londres \& New York

FRANÇOIS, Alexandre

1999 "L'illusion des classificateurs", Faits de Langues, Vol.7, n¹4, Ophrys, Paris:165-75

GRAHAM, Ian Corpus of Maya Hieroglyphic Inscriptions, Vol.7, Part.1:
Seibal, Peabody Museum of Archaeology and Ethnology, Université Harvard, Cambridge (Etats-Unis)

GRAHAM, Ian \& Eric VON EUW

$1992 \quad$ Corpus of Maya Hieroglyphic Inscriptions, Vol.4, Part.3:

Uxmal, Xcalumkin, Peabody Museum of Archaeology and Ethnology,

Université Harvard, Cambridge (États-Unis)

GRINEVALD, Colette

1999 "Typologie des systèmes de classification nominale", Faits de Langues, Vol.7, n¹4, Ophrys, Paris:101-22

GRONEMEYER, Sven

2005 document diffusé à la XIème conférence WAYEB (2006), Malmö

JONES, Christopher \& Linton SATTERTHWAITE

1982 "The Monuments and Inscriptions of Tikal: the Carved Monuments", Tikal Report $\mathrm{n}^{\circ} 33$ - Part. A, The University Museum, Université de Pennsylvanie, Philadelphie

SCHELE, Linda

1990 "The Early Classic Dynasty History of Copán- Interim Report 1989”, Copán Mosaics Project- Copan Note 70, Copán \& Austin

SCHELE, Linda \& David FREIDEL

$1990 \quad$ A Forest of Kings: The Untold Story of the Ancient Maya, William Morrow and Co, New York

SCHELE, Linda \& Matthew G. LOOPER

1996 Notebook for the XXth Maya Hieroglyphic Workshop- March 9-10, 1996, University of Texas, Austin 